\title{
Contenido mineral en hojas de la hojarasca del matorral espinoso tamaulipeco
}

\author{
Mineral content in leaf litter of Tamaulipas thorny scrub \\ Roque G. Ramírez-Lozano ${ }^{1}$, Israel Cantú-Silva ${ }^{2}$, Marco V. Gómez-Meza ${ }^{3}$, Humberto González-Rodríguez ${ }^{2 *}$ \\ ${ }^{1}$ Facultad de Ciencias Biológicas, Dpto. Alimentos, Universidad Autónoma de Nuevo León, Monterrey, NL, México \\ ${ }^{2}$ Facultad de Ciencias Forestales, Universidad Autónoma de Nuevo León, Apartado Postal 41, Linares, NL, CP. 67700 \\ ${ }^{3}$ Facultad de Economía, Universidad Autónoma de Nuevo León, Monterrey, NL, México \\ *Autor de correspondencia: humberto.gonzalezrd@uanl.edu.mx
}

Nota científica recibida: 27 de febrero de 2016 aceptada: 25 de abril de 2017

RESUMEN. El objetivo del estudio fue evaluar y comparar en espacio y tiempo la concentración de $\mathrm{Ca}, \mathrm{K}, \mathrm{Mg}, \mathrm{P}$, $\mathrm{Cu}, \mathrm{Fe}, \mathrm{Mn}$ y $\mathrm{Zn}$ en hojarasca colectada en el municipio de Linares, Nuevo León, México. Los contenidos de todos los minerales, excepto de $\mathrm{Cu}$ y $\mathrm{Zn}$, fueron diferentes estadísticamente entre sitios, meses e interacciones. El Ca varió de 23 a $43 \mathrm{~g} \mathrm{~kg}^{-1}$ en base seca, el $\mathrm{K}$ fue de 3 a 9, el $\mathrm{Mg}$ de 2 a 4 y el $\mathrm{P}$ de 0.2 a 1.1. El Cu varió de 4 a 7 , el Fe de 103 a 654, el $\mathrm{Mn}$ de 22 a 42 y el $\mathrm{Zn}$ de 12 a $25 \mathrm{mg} \mathrm{kg}^{-1}$. El contenido de $\mathrm{Mg}, \mathrm{P}, \mathrm{Cu}$ y $\mathrm{Zn}$ no es suficiente para satisfacer los requerimientos metabólicos de rumiantes.

Palabras clave: Tejido foliar, minerales, requerimientos metabólicos, rumiantes

ABSTRACT. The objective of the study was to evaluate and compare in space and time the concentration of $\mathrm{Ca}, \mathrm{K}$, $\mathrm{Mg}, \mathrm{P}, \mathrm{Cu}, \mathrm{Fe}, \mathrm{Mn}$ and $\mathrm{Zn}$ in litter collected in the municipality of Linares, Nuevo León, Mexico. The contents of all minerals, except $\mathrm{Cu}$ and $\mathrm{Zn}$, were statistically different among sites, months and interactions. Ca ranged from 23 to $43 \mathrm{~g} \mathrm{~kg}^{-1}$ dry basis, $\mathrm{K}$ was from 3 to $9, \mathrm{Mg}$ from 2 to 4 and $\mathrm{P}$ from 0.2 to 1.1 . Cu varied from 4 to 7 , Fe from 103 to $654, \mathrm{Mn}$ from 22 to 42 and $\mathrm{Zn}$ from 12 to $25 \mathrm{mg} \mathrm{kg}^{-1}$. The content of $\mathrm{Mg}, \mathrm{P}, \mathrm{Cu}$ and $\mathrm{Zn}$ is not enough to satisfy the metabolic requirements of ruminants.

Key words: Leaf tissue, minerals, metabolic requirements, ruminants

\section{INTRODUCCIÓN}

En el noreste de México, la hojarasca de la vegetación del matorral espinoso tamaulipeco está compuesta por las hojas, ramas, estructuras reproductivas y componentes diversos (GonzálezRodríguez et al. 2011a, González-Rodríguez et al. 2011b). La media anual de $4500 \mathrm{~kg} \mathrm{ha}^{-1}$ de hojarasca producida en la región, puede ser una alternativa de alimento para los pequeños rumiantes, en los períodos secos y durante la escasez de forraje (Domínguez-Gómez et al. 2011).

Estudios previos reportan elevados consumos de hojas caídas por los pequeños rumiantes (Ramírez-Lozano 2012). Aun cuando la hojarasca es una importante fuente de elementos minerales, que pueden ser utilizados por rumiantes en pastoreo (Kumar y Tewari 2014), hay carencia de información sobre las evaluaciones espacio-temporales del perfil de minerales en las hojas caídas en regiones semiáridas (López-Hernández et al. 2013). Se sabe que los herbívoros bajo condiciones de pastoreo obtienen los minerales de las plantas que consumen (Keba et al. 2013, Gul et al. 2016, Roukos et al. 2017). Un consumo inadecuado de minerales, provoca baja en la productividad (García-Vaquero et al. 2011). Por lo que el objetivo del estudio fue evaluar y comparar en el espacio y el tiempo la concentración mineral en la hojarasca proveniente del matorral espinoso tamaulipeco (MET) del noreste de México. 


\section{MATERIALES Y MÉTODOS}

\section{Área de estudio y colecta de hojarasca}

El estudio se llevó a cabo en dos sitios sin disturbio localizados en el municipio de Linares, Nuevo León, México. La selección de los sitos se debió a que son grandes productores de hojarasca (González-Rodríguez et al. 2011a). La vegetación de los sitios corresponde a matorral espinoso tamaulipeco. El primer sitio (Campus) se ubicó dentro del Campus Experimental de la Facultad de Ciencias Forestales de la Universidad Autónoma de Nuevo León, localizada a $24^{\circ} 46^{\prime} 43^{\prime \prime}$ LN y $99^{\circ} 31^{\prime} 39^{\prime \prime}$ LO; con altitud de $370 \mathrm{msnm}$, temperatura media anual de $22{ }^{\circ} \mathrm{C}$ y precipitación anual acumulada de alrededor de $800 \mathrm{~mm}$, suelos tipo Vertisoles. El segundo sitio (Crucitas) se ubicó en el Ejido Crucitas, localizado a $24^{\circ} 46^{\prime} 35^{\prime \prime}$ LN y $99^{\circ} 41^{\prime} 44^{\prime \prime}$ LO; a una altitud de $550 \mathrm{msnm}$, temperatura media anual de $21^{\circ} \mathrm{C}$ y precipitación anual acumulada de $755 \mathrm{~mm}$, los suelos son Castañozem y Chernozem (López-Hernández et al. 2013).

El principal tipo de vegetación en los sitios de estudio se caracteriza por ser de tipo arbustiva y subarbórea. Siendo las especies más representativas Acacia amentacea DC., Castela erecta Turpin subsp texana (Torr. y A. Gray) Cronquist, Celtis pallida Torr., Parkinsonia texana var. macra (IM Johnst.) Isely, Forestiera angustifolia Torr., Cordia boissieri A. DC., Leucophyllum frutescens (Berland.) IM Johnst., Opuntia spp, Zanthoxylum fagara Sarg., Bumelia celastrina Kunth y Helietta parvifolia (A. Gray ex Hemsl) Benth (Domínguez-Gómez et al. 2013a).

Para la colecta de hojarasca, en cada sitio, se establecieron diez trampas o colectores de hojarasca $\left(1.0 \mathrm{~m}^{2}\right)$. Los colectores fueron distribuidos al azar en cada sitio (aproximadamente $2500 \mathrm{~m}^{2}$ ). Cada trampa se colocó aproximadamente a $50 \mathrm{~cm}$ sobre el nivel del suelo para interceptar la hojarasca (González-Rodríguez et al. 2011b). La colecta de la hojarasca se realizó cada $15 \mathrm{~d}$, de enero a diciembre; las dos colectas de cada mes se mezclaron en una sola para luego secarlas en una estufa de aire forzado (Lab-Line, modelo $3476{ }^{\circledR}$ ) hasta peso constante a
$65{ }^{\circ} \mathrm{C}$ por $72 \mathrm{~h}$. El contenido de hojarasca se separó de forma manual en hojas, estructuras reproductivas (flores, frutos y semillas), ramas y otros tejidos no identificados (estructuras fina, corteza, fragmento de cuerpos de insectos o heces). Las hojas se molieron en un molino Thomas Willey (Thomas Scientific Apparatus, Modelo $\left.3383{ }^{\circledR}\right)$ usando una malla No. $60(1.0 \mathrm{~mm} \times 1.0 \mathrm{~mm})$.

\section{Determinación de minerales}

El contenido de minerales de las hojas se determinó, por triplicado, para lo cual se realizó una digestión húmeda. Se tomó una muestra de $1.0 \mathrm{~g}$ para incinerarla en una mufla a $550{ }^{\circ} \mathrm{C}$ por $5 \mathrm{~h}$. Posteriormente, las cenizas se digerieron en una solución de $\mathrm{HCl}$ y $\mathrm{HNO}_{3}$ concentrado (proporción $10: 1, v / v)$, y se colocaron en matraces de aforación de $25 \mathrm{~mL}$. Las concentraciones de $\mathrm{Ca}, \mathrm{Mg}$, $\mathrm{K}, \mathrm{Na}, \mathrm{Fe}, \mathrm{Mn}$ y $\mathrm{Zn}$ se determinaron por espectrofotometría de absorción atómica con un espectrofotómetro Varian (modelo SpectrAA-200 ${ }^{\circledR}$ ); mientras que el $P$ se cuantificó con un espectrofotómetro UV-Visible Perkin-Elmer (modelo Lambda $1 \mathrm{~A}^{\circledR}$ ) (AOAC 2012).

\section{Diseño experimental y análisis estadístico}

Los datos de los minerales se analizaron bajo un diseño completamente al azar con arreglo bifactorial incluyendo los efectos de los dos sitios, fechas e interacciones (Steel y Torrie 1980). Todos los análisis estadísticos se realizaron con el paquete computacional SPSS de Windows versión 22.

\section{RESULTADOS Y DISCUSIÓN}

\section{Macrominerales}

Las muestras evaluadas en todos los meses, en los dos sitios, excepto octubre del sitio Crucitas, presentaron concentraciones de minerales superiores a $20 \mathrm{~g} \mathrm{~kg}^{-1}$ de materia seca (Tabla 1). A pesar de las variaciones en espacio y tiempo, todas las muestras presentaron cantidades suficientes de 17 a $43 \mathrm{~g}$ de $\mathrm{Ca} \mathrm{kg}^{-1}$ de materia seca para satisfacer los requerimientos de bovinos de carne, borregos, cabras y venados cola blanca en pastoreo 
Tabla 1. Contenido de macrominerales ( $\mathrm{g} \mathrm{kg}^{-1} \mathrm{MS}$ ) en la hojarasca de matorral espinoso tamaulipeco.

\begin{tabular}{|c|c|c|c|c|c|}
\hline Sitios & Meses & $\mathrm{Ca}$ & $\mathrm{K}$ & $\mathrm{Mg}$ & $\mathrm{P}$ \\
\hline \multirow[t]{12}{*}{ Campus } & Enero & 37 & 6 & 4 & 0.7 \\
\hline & Febrero & 40 & 9 & 4 & 0.7 \\
\hline & Marzo & 43 & 9 & 4 & 1.1 \\
\hline & Abril & 35 & 5 & 4 & 0.4 \\
\hline & Mayo & 34 & 3 & 3 & 0.6 \\
\hline & Junio & 29 & 4 & 4 & 0.9 \\
\hline & Julio & 29 & 5 & 4 & 0.8 \\
\hline & Agosto & 35 & 6 & 4 & 0.5 \\
\hline & Septiembre & 36 & 4 & 3 & 0.4 \\
\hline & Octubre & 36 & 6 & 3 & 0.6 \\
\hline & Noviembre & 40 & 7 & 4 & 0.6 \\
\hline & Diciembre & 40 & 7 & 4 & 0.7 \\
\hline \multirow[t]{12}{*}{ Crucitas } & Enero & 26 & 6 & 3 & 0.6 \\
\hline & Febrero & 26 & 6 & 3 & 0.6 \\
\hline & Marzo & 34 & 7 & 3 & 1.0 \\
\hline & Abril & 27 & 5 & 3 & 0.3 \\
\hline & Mayo & 20 & 5 & 2 & 0.5 \\
\hline & Junio & 22 & 6 & 3 & 0.5 \\
\hline & Julio & 22 & 6 & 2 & 0.6 \\
\hline & Agosto & 24 & 6 & 2 & 0.5 \\
\hline & Septiembre & 24 & 3 & 2 & 0.3 \\
\hline & Octubre & 17 & 5 & 2 & 0.2 \\
\hline & Noviembre & 26 & 7 & 3 & 0.7 \\
\hline & Diciembre & 25 & 6 & 3 & 0.6 \\
\hline Media total & & 30 & 6 & 4 & 0.5 \\
\hline \multirow[t]{2}{*}{ EEM } & & 1 & 0.3 & 0.1 & 0.02 \\
\hline & & alor de F c & culada y s & nificancia & \\
\hline \multirow[t]{3}{*}{ Efectos } & Sito $(A)$ & $117.5^{* * *}$ & $8.5 * *$ & $108.8 * * *$ & $4.6 * * *$ \\
\hline & Mes (B) & $2.5^{* *}$ & $16.4 * * *$ & $3.2^{* *}$ & $37.2 * * *$ \\
\hline & $A \times B$ & $2.2 * *$ & $9.4 * * *$ & $2.8 * *$ & $4.6 * * *$ \\
\hline
\end{tabular}

de 4.6, 5.1, 3.0 y $5.3 \mathrm{~g}$ de $\mathrm{Ca} \mathrm{kg}^{-1}$ de materia seca en la dieta (Ramírez-Lozano 2012). Resultados similares fueron reportados por Alvarado et al. (2012) quienes evaluaron el contenido de Ca en las hojas de ocho arbustos nativos de la flora del noreste de México, mientras que Guerrero-Cervantes et al. (2012) encontraron valores similares en hojas de arbustivas que crecen en regiones semiáridas, quienes indican que estas tienen suficiente Ca para el desarrollo óptimo del ganado y la fauna silvestre. Al parecer, el $\mathrm{pH}$ ligeramente alcalino en los suelos de estas regiones puede causar que las arbustivas contengan altos niveles de Ca (González-Rodríguez et al. 2011b).

Los rumiantes adultos en pastoreo con hojarasca, en cualquiera de los meses en los dos sitios de estudio, pueden cubrir sus requerimientos metabólicos de $\mathrm{K}$ de $6.0,6.5,4.6$ y $4.6 \mathrm{~g} \mathrm{~kg}^{-1}$ para bovinos de carne, borregos, cabras y venado cola blanca (Ramírez-Lozano et al. 2010). Resultados similares han sido reportados por Alvarado et al. (2012) y Guerrero-Cervantes et al. (2012) quienes evaluaron el contenido de $\mathrm{K}$ de especies arbustivas en regiones áridas y semiáridas.

Con respecto al $\mathrm{Mg}$, en la mayoría de los meses en los dos sitios tuvieron cantidades marginales suficientes para cubrir las necesidades metabólicas de los rumiantes adultos en pastoreo de $1.0,1.5,1.6$ y $1.6 \mathrm{~g}$ de $\mathrm{Mg} \mathrm{kg}^{-1}$ de la materia seca para bovinos de carne, borregos, cabras y venados cola blanca, respectivamente (NRC 2007). Al respecto Ghazanfar et al. (2011) al evaluar el contenido de $\mathrm{Mg}$ en hojas caídas de arbustivas en Pakistán, determinaron que cubren los requerimientos metabólicos de rumiantes adultos. Mientras que Alvarado et al. (2012) encontraron que las hojas de plantas arbustivas del noreste de México, contienen suficiente $\mathrm{Mg}$ para cubrir los requerimientos 
Tabla 2. Contenido de microminerales ( $\mathrm{mg} \mathrm{kg}^{-1} \mathrm{MS}$ ) en la hojarasca de matorral espinoso tamaulipeco.

\begin{tabular}{|c|c|c|c|c|c|}
\hline Sitios & Meses & $\mathrm{Cu}$ & $\mathrm{Fe}$ & $\mathrm{Mn}$ & $\mathrm{Zn}$ \\
\hline \multirow[t]{12}{*}{ Campus } & Enero & 7 & 243 & 41 & 16 \\
\hline & Febrero & 7 & 394 & 41 & 18 \\
\hline & Marzo & 9 & 654 & 43 & 25 \\
\hline & Abril & 7 & 343 & 42 & 15 \\
\hline & Mayo & 3 & 259 & 41 & 14 \\
\hline & Junio & 6 & 121 & 26 & 17 \\
\hline & Julio & 7 & 144 & 28 & 16 \\
\hline & Agosto & 6 & 170 & 29 & 16 \\
\hline & Septiembre & 6 & 194 & 33 & 13 \\
\hline & Octubre & 6 & 204 & 34 & 14 \\
\hline & Noviembre & 7 & 260 & 33 & 23 \\
\hline & Diciembre & 7 & 395 & 34 & 22 \\
\hline \multirow[t]{12}{*}{ Crucitas } & Enero & 6 & 128 & 33 & 17 \\
\hline & Febrero & 6 & 141 & 30 & 17 \\
\hline & Marzo & 7 & 240 & 35 & 19 \\
\hline & Abril & 5 & 152 & 25 & 14 \\
\hline & Mayo & 4 & 131 & 26 & 15 \\
\hline & Junio & 5 & 108 & 26 & 15 \\
\hline & Julio & 5 & 156 & 22 & 14 \\
\hline & Agosto & 5 & 184 & 26 & 15 \\
\hline & Septiembre & 5 & 171 & 25 & 15 \\
\hline & Octubre & 5 & 117 & 26 & 12 \\
\hline & Noviembre & 6 & 103 & 27 & 17 \\
\hline & Diciembre & 7 & 112 & 28 & 19 \\
\hline Media total & & 6 & 230 & 33 & 17 \\
\hline \multirow[t]{2}{*}{ EEM } & & 0.2 & 6 & 1 & 1 \\
\hline & \multicolumn{5}{|c|}{ Valor de F calculada y significancia } \\
\hline \multirow[t]{3}{*}{ Efectos } & Sito $(A)$ & $0.02 \mathrm{~ns}$ & $12.8 * * *$ & $133.7 * * *$ & $1.97 \mathrm{~ns}$ \\
\hline & Mes (B) & $4.9 * * *$ & $7.3^{* * *}$ & $11.0 * * *$ & $6.0 * * *$ \\
\hline & $A \times B$ & $1.5 \mathrm{~ns}$ & $6.1^{* * *}$ & $7.0 * * *$ & $1.1 \mathrm{~ns}$ \\
\hline
\end{tabular}

metabólicos de pequeños rumiantes en pastoreo.

Las muestras de hojarasca, en todos los meses en los dos sitios, registraron cantidades insuficientes de $\mathrm{P}$ para cubrir las necesidades metabólicas de bovinos de carne, borregos, cabras y venados cola blanca en pastoreo de 2.3, 2.7, 2.8 y $2.6 \mathrm{~g} \mathrm{de} \mathrm{P} \mathrm{kg}^{-1}$ de materia seca (Ramírez-Lozano 2012). Por otro lado, elevados niveles de Ca comparados con los de $\mathrm{P}$ provocó una inusual relación desproporcionada de 60:1. Sin embargo, los pequeños rumiantes que ramonean como las cabras y el venado cola blanca pueden mantener estas relaciones de $\mathrm{Ca}: \mathrm{P}$ sin que se vea afectado el metabolismo del P (Ramírez-Lozano 2012).

\section{Microminerales}

El $\mathrm{Cu}$ fue deficiente en todos los meses y sitios, por lo que no satisface las necesidades metabólicas de bovinos de carne (Tabla 2), borre- gos, cabras y venados cola blanca en pastoreo de 11 , 9,9 y $9 \mathrm{mg}$ de $\mathrm{Cu} \mathrm{kg}{ }^{-1}$ de materia seca, en las dietas (Ramírez-Lozano 2012). Bajas concentraciones de Cu también han sido reportadas en regiones semiáridas (Guerrero-Cervantes et al. 2012, Alvarado et al. 2013). Los niveles de $\mathrm{Cu}$ en las plantas varían de 6 a $9 \mathrm{mg} \mathrm{kg}^{-1}$ en la materia seca, pueden deberse al $\mathrm{pH}$ ligeramente alcalino de los suelos de estas regiones (González-Rodríguez et al. 2011b, LópezHernández et al. 2013). El alto contenido de fibra en las muestras de hojarasca (Rodríguez-Santillán et al. 2015), especialmente en invierno y primavera pudieron haber reducido las concentraciones de $\mathrm{Cu}$ (Domínguez-Gómez et al. 2013b).

En todos los meses y sitios, todas las muestras de hojarasca tuvieron concentraciones de Fe adecuadas para satisfacer los requerimientos metabólicos de bovinos de carne, borregos, cabras y venados cola blanca adultos en pastoreo de $45 \mathrm{mg}$ de 
Fe $\mathrm{kg}^{-1}$ en la materia seca de la dieta (RamírezLozano 2012). Resultados similares han sido reportados por Alvarado et al. (2013) y Ramírez-Lozano et al. (2010) quienes evaluaron el contenido de $\mathrm{Fe}$ en las hojas de plantas arbustivas, que crecen en regiones semiáridas de México. Dependiendo de la estación y precipitación, los contenidos de $\mathrm{Fe}$ en los suelos de los sitios de estudio fluctúan de 2.5 a $14.61 \mathrm{mg} \mathrm{kg}^{-1}$ (López-Hernández et al. 2013). De acuerdo a Salgado-García et al. (2013), contenidos de $\mathrm{Fe}$ en el rango de 2.5 a 4.5 se consideran marginal, y mayores de 4.5 son adecuados.

Los bovinos de carne, borregos, cabras y venados cola blanca requieren $30 \mathrm{mg}$ de $\mathrm{Mn} \mathrm{kg}^{-1}$ en la materia seca de la dieta (Ramírez-Lozano 2012). En este estudio, solo las muestras de hojarasca del sitio Campus, con excepción de los meses de junio, julio y agosto, tuvieron contenidos adecuados de $\mathrm{Mn}$ para satisfacer las necesidades metabólicas. $\mathrm{Se}$ ha documentado que el $\mathrm{Mn}$ en el suelo presenta variaciones estacionales de asimilabilidad debido a la precipitación y los micro organismos (Salgado y Núñez 2012). Si el contenido de Mn si es menor a $1.0 \mathrm{ppm}$, es deficiente en el suelo, por lo contrario si es mayor a $1.0 \mathrm{ppm}$ es adecuado (Salgado-García et al. 2013). Los contenidos de $\mathrm{Mn}$ en el suelo en los sitios de estudio están por encima de las 5 ppm (López-Hernández et al. 2013). Bajos niveles de $\mathrm{Mn}$ en las hojas de plantas arbustivas que crecen en el norte de México han sido reportados por Guerrero-Cervantes et al. (2012). También se reportan elevados contenidos de $\mathrm{Ca}$ en las arbustivas mexicanas.

Las muestras de hojarasca, en todos los meses y sitios tuvieron cantidades insuficientes de $\mathrm{Zn}$ para satisfacer los requerimientos metabólicos de rumiantes en pastoreo de 35, 27, 45 y $45 \mathrm{mg}$ de $\mathrm{Zn} \mathrm{kg}^{-1}$ de materia seca de la dieta de bovinos de carne, borregos, cabras y venados cola blanca, respectivamente (Ramírez-Lozano et al. 2010). Al respecto Ramírez-Lozano (2012) y Alvarado et al. (2013) reportan que la mayoría de las arbustivas, que crecen en el noreste de México y sur de Texas, EUA, no contienen $\mathrm{Zn}$ en cantidades satisfactorias para los rumiantes domésticos y silvestres. Dependiendo de la estación y régimen de precipitación, los contenidos de $\mathrm{Zn}$ en los suelos de los sitios de estudio varían de 1.13 a $4.41 \mathrm{mg} \mathrm{kg}^{-1}$ (LópezHernández et al. 2013).

Solo el $\mathrm{Ca}, \mathrm{K}, \mathrm{Mg}, \mathrm{Mn}$ (sitio Campus) y Fe en todas las muestras de hojarasca en los dos sitios tuvo suficiente contenido mineral para satisfacer los requerimientos metabólicos de rumiantes adultos. Por lo que los rumiantes que pastoreen en estos sitios deben ser suplementados con $\mathrm{Mg}, \mathrm{Cu}, \mathrm{Zn}$ y P. El elemento más limitante fue el $\mathrm{P}$; por lo que el crecimiento y el desarrollo óseo de los rumiantes que consumen la hojarasca del matorral espinoso tamaulipeco, se puede ver limitado.

\section{AGRADECIMIENTOS}

Al Consejo Nacional de Ciencia y Tecnología (CONACYT), por el financiamiento del proyecto CONACYT, clave 250732. A los tres revisores anónimos por enriquecer y hacer observaciones críticas y constructivas al presente escrito.

\section{LITERATURA CITADA}

Alvarado MS, Ramírez-Lozano RG, González-Rodríguez H, Cantú-Silva I, Gómez-Meza MV, Cotera-Correa M, et al. (2012) Macro mineral content in five shrubs browsed by white-tailed deer (Odocoileus virginianus), northeastern Mexico. Journal of Animal and Veterinary Advances 11: 4182-4189.

Alvarado MS, Ramírez-Lozano RG, González-Rodríguez H, Cantú-Silva I, Gómez-Meza MV, Cotera-Correa $\mathrm{M}$, et al. (2013) Trace elements in native shrubs consumed by white-tailed deer (Odocoileus virginianus) in northeastern Mexico. Journal of Applied Animal Research 41: 277-284.

AOAC (2012) Official Methods of Analysis. 19th Edition. Association of Official Analytical Chemists. Gaithersburg, Maryland, USA. 2200p. 
Domínguez-Gómez TG, Guerrero-Cervantes M, Cerrillo-Soto MA, Juárez-Reyes AS, Ramírez Lozano RG, González Rodríguez H, et al. (2011) Polyethylene glycol influence on in vitro gas production parameters in four native forages consumed by white-tailed deer. Journal of Forest and Environmental Sciences 17: 21-32.

Domínguez-Gómez TG, González-Rodríguez H, Ramírez-Lozano RG, Estrada-Castillón AE, Cantú-Silva I, Gómez-Meza MV, et al. (2013a) Diversidad estructural del matorral espinoso Tamaulipeco durante las épocas seca y húmeda. Revista Mexicana de Ciencias Forestales 4: 106-122.

Domínguez-Gómez TG, González-Rodríguez H, Ramírez-Lozano RG, Cantú-Silva I, Gómez-Meza MV, CantúAyala CM, et al. (2013b) Nutritional profile of shrub species growing at northeastern Mexico. International Journal of Bio-resource and Stress Management 4: 1-8.

García-Vaquero M, Miranda M, López-Alonso M, Castillo C, Benedito JL (2011) Evaluation of the need of trace minerals supplementation in intensively reared beef cattle. Livestock Science 137: 273-277.

Ghazanfar S, Latif A, Mirza IH, Nadeem MA (2011) Macro-minerals concentrations of major fodder tree leaves and shrubs of district Chakwal, Pakistan. Pakistan Journal of Nutrition 10: 480-484.

González-Rodríguez H, Domínguez-Gómez TG, Cantú-Silva I, Gómez-Meza MV, Ramírez-Lozano RG, PandoMoreno M, et al. (2011a) Litterfall deposition and leaf litter nutrient return in different locations at Northeastern Mexico. Plant Ecology 212: 1747-1757.

González-Rodríguez H, Cantu-Silva I, Ramírez-Lozano RG, Gómez-Meza MV, Sarquis-Ramírez JI, Coria-Gil $\mathrm{N}$, et al. (2011b) Xylem water potentials of native shrubs from Northeastern Mexico. Acta Agriculturae Scandinavica, Section B - Plant Soil Science 61: 214-219.

Guerrero-Cervantes M, Ramírez RG, Gonzalez-Rodriguez H, Cerrillo-Soto A, Juárez-Reyes AS (2012) Mineral content in range forages from north Mexico. Journal of Applied Animal Research 40: 102-107.

Gul B, Islam M, Ahmad S, Gul S (2016) Aboveground biomass and concentration of nutrients in semiarid rangeland plant species: Influence of grazing and soil moisture. Фyton Revista Internacional de Botánica Experimental 85: 94-99.

Keba HT, Madakadze IC, Angassa A, Hassen A (2013) Nutritive value of grasses in semi-arid rangelands of Ethiopia: Local experience based herbage preference evaluation versus laboratory analysis. AsianAustralasian Journal of Animal Sciences 26: 366-377.

Kumar S, Tewari LM (2014) Patter of litter fall and litter decomposition in a Quercus leuocotrichophora A. Camus forest in Kumaun Himalaya. International Journal of Biodiversity and Conservation 6: 108-114.

López-Hernández JM, González H, Ramírez RG, Cantú I, Gómez MV, Pando M, et al. (2013) Litterfall production and potential return of nutrients in three sites of the state of Nuevo Leon, Mexico. Polibotánica 35: 41-64.

NRC (2007) Nutrient requirements of small ruminants: sheep, goats, cervids, and new world camelids. National Academy of Sciences. National Research Council. Washington, USA. 384p.

Ramírez-Lozano RG (2012) Alimentación del venado cola blanca: biología y ecología nutricional. Editorial Palibrio. EUA. 368p.

Ramírez-Lozano RG, González-Rodríguez H, Gómez-Meza MV, Cantú-Silva I, Uvalle-Sauceda JI (2010) Spatio-temporal variations of macro and trace mineral contents in six native plants consumed by ruminants at northeastern Mexico. Tropical and Subtropical Agroecosystems 12: 267-281. 
Rodríguez-Santillán P, Guerrero-Cervantes M, Ramírez-Lozano RG, Bernal-Barragán H, González-Rodríguez $H$, Juárez-Reyes AS (2015) Nutritional profile of leaf litterfall as feed resource for grazing animals in semiarid regions. Life Science Journal 12: 54-61.

Roukos CN, Kyriazopoulos AP, Abraham EM, Parissi ZM, Karatassiou M (2017) Nutritional quality of herbaceous vegetation in a Phryganic Mediterranean ecosystem during the grazing period. Notulae Botanicae Horti Agrobotanici Cluj-Napoca 45: 149-156.

Salgado GS, Núñez ER (2012) Manejo de fertilizantes y abonos orgánicos. Colegio de Postgraduados-Mundi Prensa. México. 146p

Salgado-García S, Palma-López DJ, Castelán-Estrada M, Lagunes-Espinoza LC, Ortiz LH (2013) Manual para el muestreo de suelos, plantas y aguas e interpretación de análisis para la producción sostenible de alimentos. Grupo MASCAÑA-LPI-2: AESS. Colegio de Postgraduados-Campus Tabasco. Tabasco, México. 101p

Steel RGD, Torrie JH (1980) Principles and procedures of statistics. A biometrical approach. 2nd Edition. McGraw-Hill. New York, USA. 632p 
